\title{
Centralized Pain: Status Review and Medical Management
}

\section{Ihsan F Shanti ${ }^{1}$, Bilal F Shanti ${ }^{2 *}$, Ali Otom ${ }^{3}$, Ali Al Rjoub ${ }^{4}$ and Khalil Al-Abbadi ${ }^{5}$}

${ }^{1}$ The American Clinic for Interventional Pain and Spine, Amman, Jordan

${ }^{2}$ Omnia Pain Consultants, Pain Medicine, Phoenix, Arizona, USA

${ }^{3}$ Royal Specialty Center for Spine and Musculoskeletal Disorders, Amman, Jordan

${ }^{4}$ Department of Physical Medicine and Rehabilitation, Al Bashir Hospital, Amman,

Jordan

${ }^{5}$ Senior Consultant, Physical Medicine and Neurorehabilitation, Amman, Jordan

*Corresponding Author: Bilal F Shanti, Omnia Pain Consultants, Pain Medicine, Phoenix, Arizona, USA.

DOI: 10.31080/ASNE.2020.03.0205
Received: June 20, 2020

Published: July 30, 2020

(C) All rights are reserved by Bilal F Shanti., et al.

\footnotetext{
Abstract

Centralized pain is non-fatal disorder linked to permanent changes and chaotic imprinting of pain inputs within the central nervous system. This occurs without obvious logical reasons that we know of. This persistent heightened alert of the nervous system is very exhausting to the patients and their families. The magnitude of other symptom comorbidities makes this pain entity even more challenging to diagnose and treat. Fortunately, recent research discoveries have introduced a few theories such the concept of glial activation and neuroinflammation as having some plausible role in this complex syndrome. This review manuscript will seek to explain and simplify some of the non-procedural clinical medical management options available to date, as tools for us, clinicians, to fight this painful entity.

Keywords: Centralized Pain; Central Nervous System (CNS); Amino Acids
}

\section{Introduction}

For over 50 years, clinicians have called persistent pain that is a result of a direct insult to the central nervous system (CNS), trauma, infection, cerebrovascular accident, diseases such as multiple sclerosis or Parkinson's disease; central pain or central pain syndrome [1].

More recently, there has been an explosion of research into other pain neurobiology and sources that imprint in the CNS [2].

Centralized pain, in our opinion, is one of the most challenging clinical pain syndromes to manage. This chronic debilitating pain can possibly linger for as long as the patient lives. Early intervention and a quick plan of action are essential. Treating this syndrome requires more than writing a prescription. A thorough understanding of the cascade and pathophysiology of centralized pain is in order.

Development of centralized pain: Review of theories

Centralized pain develops after an unresolved initial insultwhen acute pain becomes chronic typically about 3 months or later [4]. After the initial peripheral pain insult, inflammatory mediators from the site are transmitted to the spinal cord and CNS. In the brain, there is activation of Microglial cells which results in nerve 
inflammation. Neuron hyper-excitability, known as central sensitization, takes place [4]. This inflammation causes release of Glutamate, (a neurotransmitter and neurotoxin) and other excitatory amino acids. This in turn causes tissue destruction in the CNS (Figure 1) [3]. The inflamed, hyper excited tissue causes excess efferent signals manifested by allodynia and hyperalgesia. Excess sym- pathetic discharge occurs and hypothalamic-pituitary stimulation increases adrenal hormone production [4]. The CNS attempts to heal itself and eliminate this pathologic process [5,6]. This attempt to self-repair or reshaping of CNS tissue is referred to as neuroplasticity [7]. The ultimate manifestation of this pain is a complex chronic catastrophic clinical disease called "centralized pain" [3].

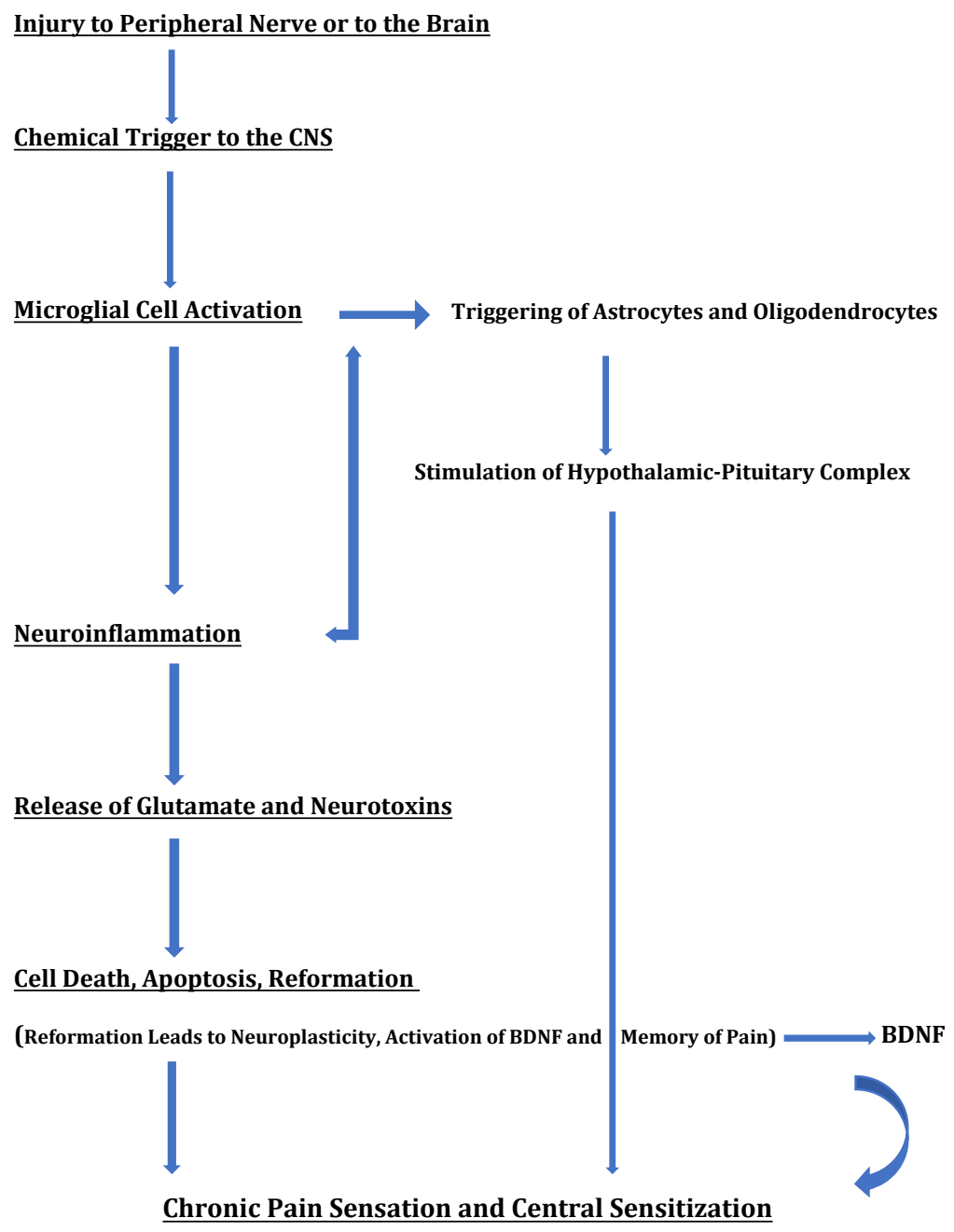

Figure 1 


\section{Medical management of centralized pain}

The clinical question that imposes itself is how does the clinician manage such a complex pain process? After a thorough review of the literature, there is presently no general consensus on the treatment of centralized pain. It is thus imperative to understand the biomolecular cascade of the glial and inflammatory pain pathways that involve this complex pain entity. This will enable the clinician to address each step accordingly and be mindful of the quick transition of primary benign pain to a secondary diagnosis of centralized pain [4]. Because there are no treatment guidelines, centralized pain is mostly treated by 'trial and error' rather than in a regimented approach. It is our opinion that each case may vary clinically depending on many factors, including age, general health, patient pain experience, genetic factors, psychological aspects, family support, smoking status, access to healthcare, and provider clinical experience. In general, it is always safe to start with formulating a long term plan of management. Centralized pain requires a systematic interdisciplinary combined therapy that may include pharmacological approach combined with an essential in-depth patient education. The provider, in turn, should be open-minded to referring the patient to other specialists for consultation and not assume a solitary role of "know-it-all".

The management of centralized pain includes medical, educational, pharmacological, psychological, alternative and oriental, nutritional, physical and rehabilitative, interventional, and surgical management options. The management options will differ according to the pain type, its location, the disease it is associated with, chronicity, and multiple other factors. In this article, we will only emphasize on a few options of treatments as many have been covered elsewhere in literature.

\section{Medical/pharmacological management}

The WHO three pain step ladder remains the gold standard of pain treatment. Albeit old and possibly outdated, it serves the primary purpose to teach us to "start low and proceed slow" in treating any general pain condition. But in the case of centralized pain, it is important to be very prompt in treating this entity from the get-go.

\section{NSAIDS and COX-2 inhibitors}

Since inflammation is a primary initiator in the cascade of centralized pain, it is always safe to start with Non-Steroidal Anti-Inflammatory Medications (NSAIDS). If used for a long time, many are hard on the gastro-intestinal (GI) system and carry cardiac, neurological and renal untoward effects. In our opinion, NSAIDS use should be limited to no more than one week provided there are no contraindications. The alternative is COX-2 Inhibitors such as Celecoxib that spares platelets breaking and has lesser GI and cardiac side effects [8]. A few years ago Coxibs were removed from the market because of serious side effects. The authors also promote the use of the spice Turmeric as a strong anti-inflammatory diet either in powder form or in capsules mixed with black pepper for optimal absorption.

Anticonvulsants, centrally-acting muscle relaxants and others

Anticonvulsants use in centralized pain has been recommended as beneficial. They are thought to act by increasing GABA-mediated inhibition, decreasing the abnormal neuronal hyperexcitability by modulating sodium and calcium channels or by inhibiting excitatory amino acids actions [9]. Both first generation anticonvulsants (e.g. valproate, carbamazepine, and benzodiazepines) and second generation drugs (gabapentin, pregabalin, topiramate, and lamotrigine) are used. These drugs were found to be as effective as amitriptyline [9]. Lamotrigine can be used as an adjunctive therapy along with second generation anticonvulsants drugs [10].

When using benzodiazepines and opioids simultaneously, clinicians should be mindful of potential side effects including respiratory depression.

Tizanidine is an alpha (2)-adrenergic receptor agonist. It is used in spasticity associated with centralized pain. It was described as "very useful" for these patients and should be titrated to effect [11]. The authors recommend its use at night time as we promote better functionality as one of the pain control goals.

Baclofen is a GABA receptor agonist, specifically of the $\mathrm{GABA}_{\mathrm{B}}$ receptors. It acts on spinal and supraspinal sites. Intrathecal baclofen was found to provide effective long-term treatment of spasticity in multiple sclerosis and other centralized pain. It was found to increase the quality of lifestyle and functional independence in appropriately selected cases [12]. In a matched cohort study, longterm treatment with intrathecal compared with oral baclofen was associated with reduced spasm frequency and severity as well as greater dose stability [13].

Dantrolene is a postsynaptic muscle relaxant. It deceases the excitation-contraction coupling in muscle cells. By opposing ry- 
anodine receptors, it inhibits $\mathrm{Ca}^{2+}$ ions release from sarcoplasmic reticulum stores [14]. Dantrolene and other agents such as prazepam (a prodrug for desmethyl-diazepam), threonine (an essential $\alpha$-amino acid that is used in the biosynthesis of proteins) and cannabinoids have been used with some variable degree of success in some centralized pain disease such as for multiple sclerosis but further research is needed [15]. Other agents such as tolperisone (centrally acting muscle relaxant, a piperidine derivative), botulinum toxin (especially type A) may also have a use in these conditions but plans are currently underway for a better coordinated effort of treatment approach [16]. Botulinum toxin A works by blocking nociceptor transduction and reducing neurogenic inflammation. It inhibits neural substances and neurotransmitters. It prevents the peripheral and central sensitization and may be used as adjuvants when other therapy is less effective [17]. But Cochrane review published in 2016 concluded that botulinum toxin A did not improve phantom limb pain intensity during the six months of follow-up compared with lidocaine/methylprednisolone [18].

\section{Clonidine}

In a qualitative analysis of nearly thirty clinical studies, there was some evidence to suggest that clonidine administered through epidural, intrathecal and local/topical route may be effective in chronic pain conditions where neuropathy is a predominant component [19]. Direct alpha2 adrenoceptor agonists, such as clonidine and dexmedetomidine, are used successfully in pain states with increased sympathetic nervous activity. These cause antinociception due to postsynaptic inhibition of spinothalamic projection neurons, presynaptic inhibition at CNS termination of primary sensory neurons, presynaptic inhibition of brainstem noradrenergic neurons and a generalized decrease in CNS sympathetic activity [20]. Their non-systemic use has been favored given their sedative side effects [20].

\section{Tricyclic antidepressants (TCAs)}

TCAs such as amitriptyline, nortriptyline, protriptyline, imipramine, doxepin, and desipramine and tetracyclic antidepressants (amoxapine and maprotiline) role in treating centralized pain has been a traditional option given the limited choice at that time. $\mathrm{Al}$ though TCAs inhibit reuptake of serotonin and norepinephrine, two key neurotransmitters in the pain modulation pathway, their long term use is poorly tolerated because of systemic side effects [21]. In addition, the use amitriptyline in phantom limb pain, the prototype of centralized pain was disappointing [18]. In a recent
Cochrane database review, the general opinion was that amitriptyline should continue to be used as part of the treatment of simple neuropathic pain, but only a minority of people will achieve satisfactory pain relief [22].

Selective serotonin reuptake inhibitors (SSRIs), Noradrenaline dopamine reuptake inhibitors (NDRIs) Norepinephrine reuptake inhibitor (NRI), and Selective noradrenergic reuptake inhibitors (SNRIs)

Since the pharmacological treatment of centralized pain encompasses the use of medications targeting different 'desensitizing' mechanism, combined therapy may prove more beneficial than monotherapy [23]. NDRIs such as bupropion and NRIs such as tapentadol (also works as a mu opioid receptor agonist) have been abundantly used with beneficial results. SSRIs such as citalopram, fluoxetine, and paroxetine and SNRIs such as duloxetine, venlafaxine and milnacipran also have been used with lower incidence of side effects compared to the traditional TCAs [24] but existing data failed to show which has the best clinical profile and outcome [24]. A meta-analytical clinical study published in 2012 on the role of anti-depressants as analgesics concluded that SNRIs were superior to SSRIs in treatment of neuropathic pain [25]. Another study confirmed that evidence does not support an analgesic effect of SSRIs but found them to have beneficial effects on well-being in several chronic pain conditions [26]. A study with special emphasis on duloxetine for treatment of fibromyalgia confirmed that SNRIs have a more consistent analgesic benefit than SSRIs [27].

\section{Intravenous ketamine and lidocaine}

The role of ketamine is becoming increasingly pivotal in the treatment of centralized pain. A study advocated the cautious use of IV ketamine in the hands of experienced providers. It outlined the outpatient use of ketamine with subanesthetic doses of in intravenous form mixed with lidocaine, propofol, and augmented by magnesium sulphate for the treatment of severe intractable pain syndromes [28]. Ketamine is an ideal anesthetic and analgesic. It works by antagonism of NMDA receptors; sites that are crucial for neuronal communication [29]. In centralized pain, the amide local anesthetic lidocaine, through its active metabolite, monoethylglycinexylidide, interacts with central and peripheral voltage-gated $\mathrm{Na}^{+}$channels in the cell membrane intracellular side [28]. IV lidocaine use for centralized pain should be carried in a controlled and monitored setting given its potential toxicity if it exceeds its allowable dose [29]. A randomized double-blind, cross-over study out of 
Sweden looked into the role of IV ketamine and lidocaine on pain after spinal cord injury. It showed that ketamine but not lidocaine showed a significant analgesic effect in patients with neuropathic pain after spinal cord injury [29].

In our review of the published data, there was an unclear role of other NMDA antagonists such as phencyclidine, methoxetamine, and nitrous oxide. Studies involving dextromethorphan in humans with centralized pain remain unclear although they may be promising in rodents [30].

\section{Ziconotide}

Ziconotide is a synthetic peptide analog of the omega-conotoxin derived from marine snail Conus Magus. It belongs to a new class of agents, the N-type calcium channel blockers and can only be used through intrathecal administration. It provides synergistic role to morphine. When blocking calcium channels, it suppresses the ectopic abnormal firing from the injury site or dorsal root ganglia decreasing the synaptic transmission needed to create and sustain chronic pain [31]. It is reported to have a narrow therapeutic window because of substantial CNS side effects and only can be used in a small subset of patient with severe and intractable pain [32]. It is the only non-opioid intrathecal analgesic that is approved by the US FDA for the treatment of refractory chronic pain [33].

\section{Opioids}

Centralized pain is unfortunately a tragic 'curse' that befalls on the patient. It is our professional opinion that the provider should explore any and more than one modality to control the patient's pain. Adjuvant therapy is paramount and depends on the existing type and stage of centralized pain and many other factors. The provider should be mindful of the potential side effects and be vigilant to any changes in the patient's health, hemodynamics, and behavior. We encourage that the provider perform a pre-treatment pharmacogenetic testing to aid in the use of appropriate pain medications. A trial of opioids should be considered if other adjuvant treatments fail. Counseling and urine drug toxicity testing should be done to ensure compliance. A thorough and 'obsessive' documentation of the type of pain, physical examination, the indication for use of opiates, and the plan of action is essential.

Consultation with different specialists may be warranted. Opioid rotation should be carried as deemed necessary. In 2 published articles, these legitimized the use of ultra-high dose if and when needed, as long as there is very good documentation to support this indication in today's age of CDC opioid regulations [34,35]. The choice of opiate depends on the case in question, provider and patient comfort and experience, and many other factors that we cannot delve in this article. The authors are very comfortable with the use of methadone for centralized pain. It has the beneficial NMDA antagonist effect. In the US, it is cheap, available and easy to titrate if the provider is familiar with it. But a Cochrane review found that methadone provided very limited evidence of its efficacy in chronic non-cancer pain [36]. Other pain medications come in many strengths and preparations that will suit your patient needs.

\section{Physical activity and exercise}

A Cochrane Database Review was published and included 264 studies (19,642 participants). It examined exercise versus no exercise/minimal intervention in adults with chronic pain. The quality of the evidence examining physical activity and exercise for chronic pain is low. This is largely due to small sample sizes and potentially underpowered studies. Pain conditions included rheumatoid arthritis, osteoarthritis, fibromyalgia, low back pain, intermittent claudication, dysmenorrhea, mechanical neck disorder, spinal cord injury, post-polio syndrome, and patellofemoral pain [37].

Another study demonstrated there was a dysfunctional response of patients with chronic pain and aberrations in central pain modulation to exercise. This indicated that exercise therapy should be individually tailored with emphasis on prevention of symptom flares [38].

In chronic pain patient with complex regional pain syndrometype (CRPS) I, it was found that physical and occupational therapy is essential [39]. Patients should be expressly encouraged to use the affected extremities, even if this is associated with a transient increase in pain and exacerbation of visible symptoms, which does not indicate deterioration [40].

In CRPS, pain-exposure physical therapy (PEPT) is a progressive loading physical exercise program and management of painavoidance behavior. It initially starts with graded exposure in vivo (GEXP) to reduce irrational disease-related fears (e.g. worsening by movement) and identifies the most "dangerous or threatening" practice tasks. These will then to be faced step by step by the patient until fear and anxiety is reduced [41]. 
In our review of the published data, the majority of publications conclude the provider and patient should discuss the patient tolerability of activity and exercise. One known conventional theory is that muscles are quick to become weak in case not used. That can affect, core stabilization and balance and may expose the patient to falls and other unwanted effects.

\section{Acupuncture, massage, meditation, and imagery}

Electro-acupuncture (EA) is reported to effectively relieve the centralized pain. EA treatments at different electrical frequencies may exert its effects by inhibiting brain neuronal apoptosis and aberrant astrocyte activation in the brain [42]. EA analgesia is likely associated with its counter-regulation to spinal glial activation [43]. A prospective, partially-blinded, controlled, randomized clinical trial found that acupuncture significantly improved symptoms of fibromyalgia, not restricted to pain relief but was most significant for fatigue and anxiety as well [44].

A Non-randomized, pre-post pilot study in multiple sclerosis showed that massage was a safe and beneficial intervention for management of fatigue and pain. There was also a correlation between decreasing fatigue and pain with improvement in quality of life [45]. In a randomized control clinical trial in patient with chronic pain from spinal cord injury, chronic pain and fatigue were significantly reduced in the massage group when assessed at the end of 5 weeks with large effect sizes [46].

A study reviewed thirty clinical trials involving adults with chronic pain and found that a low-quality evidence that mindfulness meditation is associated with a small decrease in pain compared with all types of controls [47].

The mind body theory may be a valid one. It may not be the case for chronic debilitating pain. Mind-body approaches include progressive muscle relaxation, meditation, laughter, mindfulness based approaches, hypnosis, guided imagery, yoga, biofeedback and cognitive behavioral therapy. Studies have shown that mindbody approaches can be effective in various conditions associated with chronic pain, however levels of evidence vary [48].

In patients with phantom limb pain, there were reports on the efficacy of mirror therapy. The basic idea is when the brain observes the intact limb moving, the reflection gives the brain visual and mental feedback it wasn't receiving prior to that [48].

\section{Conclusion}

Centralized pain is one of the most challenging clinical pain entities to treat. We should be thankful and have the privilege of practicing medicine and have many options available for our patients. There is no excuse for our ignorance.

\section{Conflict of Interest}

All the authors disclose they have no conflict of interest.

\section{Bibliography}

1. Jay W. "Classic central pain syndrome: review of neurologic causes of pain”. Practical Pain Management 12.7 (2012): 4863.

2. Henry DE., et al. "Central nervous system reorganization in a variety of chronic pain states: a review". PM R 3.12 (2011): 1116-1125.

3. Tennant F. "The Critical Necessity to Diagnose Pain That Is Centralized". Practical Pain Management 12.3 (2012).

4. Tennant F. "Glial Cell Activation and Neuroinflammation: How They Cause Centralized Pain”. Practical Pain Management 14.5 (2014).

5. May A. "Chronic pain may change the structure of the brain". Pain 137.1 (2008): 7-15.

6. Latremoliere A and Woolf CJ. "Central sensitization: a generator of pain hypersensitivity by central neural plasticity". Journal of Pain 10.9 (2009): 895-926.

7. Urban MK. "COX-2 specific inhibitors offer improved advantages over traditional NSAIDS”. Orthopedics 23 (2000): S761-764.

8. Finnerup NB. "Anticonvulsants in central pain". Expert Opinion on Pharmacotherapy 3.10 (2002): 1411-1420.

9. Kim JS. "Post-stroke pain". Expert Review of Neurotherapeutics 9.5 (2009): 711-721.

10. Malanga G. "Update on Tizanidine For Muscle Spasticity and Emerging Indications". Expert Opinion on Pharmacotherapy 9.12 (2008): 2209-2215.

11. Natale M. "Long-term effects of intrathecal baclofen in multiple sclerosis". Clinical Neurology and Neurosurgery 143 (2016): 121-125.

12. McCormick ZL. Intrathecal Versus Oral Baclofen: A Matched Cohort Study of Spasticity, Pain, Sleep, Fatigue, and Quality of Life". PM\&R 8.6 (2016): 553-562. 
13. Zucchi, R., et al. "The sarcoplasmic reticulum $\mathrm{Ca}^{2+}$ channel/ryanodine receptor: modulation by endogenous effectors, drugs and disease states". Pharmacological reviews 49.1 (1997): $1-51$.

14. Shakespeare DT., et al. "Anti-spasticity agents for multiple sclerosis". The Cochrane Database of Systematic Reviews 4 (2000): CD001332.

15. Gold R., et al. "Advances in the management of multiple sclerosis spasticity: multiple sclerosis spasticity guidelines". Expert Review of Neurotherapeutics. 13 (2013): 55-59.

16. Intiso D., et al. "Botulinum Toxin Type A for the Treatment of Neuropathic Pain in Neuro-Rehabilitation". Toxins (Basel). 7.7 (2015): 2454-2480.

17. Alviar MJ., et al. "Pharmacologic interventions for treating phantom limb pain". The Cochrane Database of Systematic Reviews 10 (2016): CD006380.

18. Kumar A., et al. "Clonidine for management of chronic pain: A brief review of the current evidences". Saudi Journal of Anaesthesia 8.1 (2014): 92-96.

19. Motsch J., et al. "Alpha 2-adrenergic agonists. Use in chronic pain--a meta-analysis". Schmerz 11.5 (1997): 339-344.

20. Mease PJ. Further strategies for treating fibromyalgia: the role of serotonin and norepinephrine reuptake inhibitors". American Journal of Medicine 122 (2009): S44-55.

21. Moore RA., et al. "Amitriptyline for neuropathic pain in adults". The Cochrane Database of Systematic Reviews 6.7 (2015): CD008242.

22. Nijs J., et al. "Treatment of central sensitization in patients with 'unexplained' chronic pain: an update". Expert Opinion on Pharmacotherapy 15.12 (2014): 1671-1683.

23. Mika J., et al. "Neuronal and immunological basis of action of antidepressants in chronic pain - clinical and experimental studies". Pharmacology Report 65.6 (2013): 1611-21.

24. Dharmshaktu P., et al. "Efficacy of antidepressants as analgesics: a review". Journal of Clinical Pharmacology 52.1 (2012): 6-17.

25. Verdu B., et al. "Antidepressants for the treatment of chronic pain". Drugs 68.18 (2008): 2611-2632.

26. Arnold LM. "Duloxetine and other antidepressants in the treatment of patients with fibromyalgia". Pain Medicine (2007): S63-74.
27. Krusz JC. "IV Treatment of Centralized Pain and Headache". Practical Pain Management 16.8 (2016).

28. Kvarnström A., et al. "The analgesic effect of Intravenous ketamine and Lidocaine on Pain after Spinal Cord Injury". Acta Anaesthesiologica Scandinavica 48.4 (2004): 498-506.

29. Morel V., et al. "Low doses of dextromethorphan have a beneficial effect in the treatment of neuropathic pain". Fundamental Clinical Pharmacology 28.6 (2014): 671-680.

30. Prommer E. "Ziconotide: a new option for refractory pain". Drugs Today (Barc). 42.6 (2006): 369-78.

31. Schmidtko A., et al. "Ziconotide for Treatment of Severe Chronic Pain". Lancet 375.9725 (2010): 1569-1577.

32. Sanford M. "Intrathecal ziconotide: a review of its use in patients with chronic pain refractory to other systemic or intrathecal analgesics". CNS Drugs 27.11 (2013): 989-1002.

33. Tennant F. "Ultra-high Dose Opioid Therapy: Uncommon and Declining, But Still Needed". Practical Pain Management 13.4 (2013).

34. Tennant F. “Don't Flinch From Prescribing Pain Medications!”. Practical Pain Management 16.3 (2016).

35. Haroutiunian S., et al. "Methadone for chronic non-cancer pain in adults". The Cochrane Database of Systematic Reviews 11 (2012): CD008025.

36. Geneen LJ., et al. "Physical activity and exercise for chronic pain in adults: an overview of Cochrane Reviews". The Cochrane Database of Systematic Reviews 1 (2017): CD011279.

37. Nijs J., et al. "Dysfunctional endogenous analgesia during exercise in patients with chronic pain: to exercise or not to exercise?" Pain Physician 15 (2012): ES205-213.

38. Erlemans HM., et al. "Pain and reduced mobility in complex regional pain syndrome I: outcome of a prospective randomized controlled clinical trial of adjuvant physical therapy versus occupational therapy". Pain 83 (1999): 77-83.

39. Van de Meent H., et al. "Safety of "pain exposure" physical therapy in patients with complex regional pain syndrome type 1 ". Pain 152 (2011): 1431-1438.

40. Birklein., et al. "Complex regional pain syndrome: an optimistic perspective. Views and reviews". Neurology (2015): P8996. 
41. Tian GH., et al. "Electroacupuncture Treatment Alleviates Central Poststroke Pain by Inhibiting Brain Neuronal Apoptosis and Aberrant Astrocyte Activation". Neural Plasticity (2016): 1437148.

42. Zhao ZQ. "Neural mechanism underlying acupuncture analgesia”. Progress in Neurobiology 85.4 (2008): 355-375.

43. Martin DP., et al. "Improvement in fibromyalgia symptoms with acupuncture: results of a randomized controlled trial". Mayo Clinic Proceedings 81.6 (2006): 749-757.

44. Backus D., et al. "Impact of Massage Therapy on Fatigue, Pain, and Spasticity in People with Multiple Sclerosis: a Pilot Study". The International Journal of Therapeutic Massage and Bodywork 9.4 (2016): 4-13.

45. Lovas J., et al. "Managing pain and fatigue in people with spinal cord injury: a randomized controlled trial feasibility study examining the efficacy of massage therapy". Spinal Cord (2016).

46. Hilton L., et al. "Mindfulness Meditation for Chronic Pain: Systematic Review and Meta-analysis". Annals of Behavioral Medicine 51 (2017): 199-213.

47. Hassed C. "Mind-body therapies--use in chronic pain management". Australian Family Physician 14.77 (2012): 237-46.

48. Tsao J. "Current Treatments for Phantom Limb Pain". Practical Pain Management 11.5 (2011).

\section{Assets from publication with us}

- Prompt Acknowledgement after receiving the article

- Thorough Double blinded peer review

- Rapid Publication

- Issue of Publication Certificate

- High visibility of your Published work

Website: www.actascientific.com/

Submit Article: www.actascientific.com/submission.php

Email us: editor@actascientific.com

Contact us: +919182824667

Citation: Bilal F Shanti., et al. “Centralized Pain: Status Review and Medical Management”. Acta Scientific Neurology 3.8 (2020): 16-23. 